\title{
Pulmonary vasodilator use in continuous-flow left ventricular assist device management
}

\author{
Preethi Pirlamarla ${ }^{1}$, Eduardo Rame ${ }^{1}$, Charles Hoopes ${ }^{2}$, Indranee Rajapreyar $^{3}$ \\ ${ }^{1}$ Advanced Heart Failure and Transplant Cardiology, Sidney Kimmel Medical College, Thomas Jefferson University Hospital, Philadelphia, PA, USA; \\ ${ }^{2}$ Division of Cardiothoracic Surgery, Department of Surgery, School of Medicine, University of Alabama at Birmingham, Birmingham, AL, USA; \\ ${ }^{3}$ Division of Cardiovascular Disease, Department of Medicine, University of Alabama-Birmingham School of Medicine, Birmingham, AL, USA \\ Contributions: (I) Conception and design: P Pirlamarla, I Rajapreyar; (II) Administrative support: None; (III) Provision of study materials or patients: \\ None; (IV) Collection and assembly of data: None; (V) Data analysis and interpretation: None; (VI) Manuscript writing: All authors; (VII) Final \\ approval of manuscript: All authors. \\ Correspondence to: Indranee Rajapreyar, MD. 1900 University Blvd, THT 311, Birmingham, AL 35243, USA. Email: irajapreyar@uabmc.edu.
}

\begin{abstract}
Pulmonary hypertension $(\mathrm{PH})$ due to left heart disease is the most common etiology for $\mathrm{PH}$. $\mathrm{PH}$ in patients with heart failure with reduced fraction ( $\mathrm{HFrEF}$ ) is associated with reduced functional capacity and increased mortality. PH-HFrEF can be isolated post-capillary or combined pre- and post-capillary PH. Chronic elevation of left-sided filling pressures may lead to reverse remodeling of the pulmonary vasculature with development of precapillary component of $\mathrm{PH}$. Untreated $\mathrm{PH}$ in patients with HFrEF results in predominant right heart failure (RHF) with irreversible end-organ dysfunction. Management of $\mathrm{PH}-\mathrm{HFrEF}$ includes diuretics, vasodilators like angiotensin-converting enzyme inhibitors or angiotensinreceptor blockers or angiotensin-receptor blocker-neprilysin inhibitors, hydralazine and nitrates. There is no role for pulmonary vasodilator use in patients with $\mathrm{PH}-\mathrm{HFrEF}$ due to increased mortality in clinical trials. In patients with end-stage $\mathrm{HFrEF}$ and fixed $\mathrm{PH}$ unresponsive to vasodilator challenge, implantation of continuous-flow left ventricular assist device (cfLVAD) results in marked improvement in pulmonary artery pressures within 6 months due to left ventricular (LV) mechanical unloading. The role of pulmonary vasodilators in management of precapillary component of $\mathrm{PH}$ after cfLVAD is not well-defined. The purpose of this review is to discuss the pharmacologic management of $\mathrm{PH}$ after cfLVAD implantation.
\end{abstract}

Keywords: Combined pre and post-capillary pulmonary hypertension; pulmonary vasodilators; continuous-flow left ventricular assist device (cfLVAD); right heart failure (RHF)

Submitted Jun 16, 2020. Accepted for publication Jul 30, 2020.

doi: 10.21037/atm-20-4710

View this article at: http://dx.doi.org/10.21037/atm-20-4710

\section{Introduction}

Pulmonary hypertension (PH) due to left heart disease (PH-LHD), predominantly myocardial or valvular heart disease, is the most prevalent etiologic classifier for $\mathrm{PH}$. $\mathrm{PH}$ in heart failure with reduced ejection fraction (HFrEF) is associated with reduced functional capacity, increased frequency of hospitalizations and increased mortality (1). Right heart failure (RHF) is a predominant clinical feature of HFrEF with secondary $\mathrm{PH}$, and the presence of right ventricular dysfunction (RVD) portends a poor prognosis
$(2,3)$. Irreversible end-organ dysfunction develops with progression of RVD that can preclude advanced therapies including the transition to long-term mechanical circulatory support with a left ventricular assist device (LVAD).

Significant progress has been made in understanding the pathophysiology, progression and consequences of $\mathrm{PH}-$ HFrEF. The treatment strategies for PH due to HFrEF include medical therapy targeting adequate diuresis and volume management, systemic afterload reduction with oral nitrates, angiotensin-converting enzyme inhibitors or angiotensin- receptor blockers or angiotensin receptor 
blocker-neprilysin inhibitors, hydralazine and surgical or percutaneous mitral valve interventions for functional mitral regurgitation (MR) (4-6). The treatment of PH-HFrEF with pulmonary vasodilators has yielded disappointing results with heart failure(HF) exacerbation and increased mortality using endothelin-receptor agonists and prostacyclins (7). A signal towards improved functional capacity and hemodynamics with phosphodiesterase 5 inhibitors (PDE5i) in PH-HFrEF has been shown in small single-center trials (8-12). Due to lack of strong clinical data from multicenter randomized trials, PDE5i cannot be recommended for management of $\mathrm{PH}-\mathrm{HFrEF}$.

Durable continuous-flow left ventricular assist devices (cfLVAD) have changed the landscape for management of patients with end-stage systolic heart failure (ESHF). The improved survival rates with cFLVAD has led to the approval of these devices as a bridge to transplant or destination therapy. In patients with ESHF who are not candidates for heart transplantation due to lack of reversibility of $\mathrm{PH}$ despite vasodilator challenge, cfLVAD is known to reverse $\mathrm{PH}$ within 6 months of implantation (13-19). The International Society for Heart Lung Transplantation guideline update for heart transplant listing suggests adding PDE5i in patients with persistent $\mathrm{PH}$ after $\operatorname{LVAD}(20)$.

cfLVAD unloads the left ventricle with a decrease in LV end-diastolic pressure and volume, left atrial pressure, and functional MR. The mechanical unloading of the LV reduces the pulsatile load to the pulmonary circulation with reduction in pulmonary artery (PA) pressures and PVR. However, the optimal unloading of LV to achieve near normalization of left-sided filling pressures and reversal of $\mathrm{PH}$ while preventing loss of the interventricular septal contribution to right ventricular (RV) function can be challenging. In addition, patients may have persistent $\mathrm{PH}$ despite adequate mechanical unloading due to coexistent pulmonary disease and untreated obstructive sleep apnea. Hence, a multifaceted and individualized approach for management of $\mathrm{PH}$ after cfVLAD will prevent early and late RHF. In this review, we discuss the current knowledge gap in the use of pulmonary vasodilators for the management of $\mathrm{PH}$ after cfLVAD and rationale for their use.

\section{Hemodynamic definition of PH-LHD}

In 1958, Paul Wood proposed a hemodynamic classification of $\mathrm{PH}$ and described the development of $\mathrm{PH}$ due to left ventricular failure and valvular heart disease such as mitral stenosis as "passive" due to an increase in pulmonary venous pressures (21). The most recent $6^{\text {th }}$ World Symposium on $\mathrm{PH}$ proposed hemodynamic definitions for $\mathrm{PH}$ due to left-sided heart disease. Isolated post-capillary pulmonary hypertension (IpcPH) was defined as mean pulmonary artery pressure $(\mathrm{mPAP})>20 \mathrm{mmHg}$, pulmonary capillary wedge pressure $(\mathrm{PAWP})>15 \mathrm{mmHg}$ and $\mathrm{PVR} \leq 3$ wood units (WU). Combined pre and post-capillary $\mathrm{PH}(\mathrm{CpcPH})$ was defined as mPAP $>20 \mathrm{mmHg}$, PAWP $>15 \mathrm{mmHg}$ and $\mathrm{PVR} \geq 3 \mathrm{WU}(22)$.

\section{PH-HFrEF: epidemiology and prognosis}

The true estimates of $\mathrm{PH}$ in HFrEF are variable depending on the severity of heart failure, variable definitions used to define $\mathrm{PH}$, presence of comorbidities, study era, and noninvasive versus invasive methods of estimation of $\mathrm{PA}$ pressures. Most studies did not differentiate IpcPH and $\mathrm{CpcPH}$.

The prevalence of $\mathrm{PH}$ in ambulatory patients with HFrEF by invasive hemodynamic monitoring is $40-70 \%$ $(3,23,24)$. CpcPH in ambulatory and hospitalized patients with systolic heart failure is seen in approximately $40-50 \%$ respectively $(23,25)$. MR, diastolic dysfunction, and RVD were significantly worse in $\mathrm{CpcPH}$ compared to IpcPH $(23,26)$. Older age, diabetes mellitus, and atrial fibrillation were predictors of $\mathrm{PH}$ in an ambulatory cohort of HFrEF patients referred for invasive hemodynamic monitoring.

The presence of $\mathrm{PH}$ and worsening of PA pressures increase the risk of decompensated heart failure and cardiac death by two-fold (24). Miller et al. further prognosticated $\mathrm{PH}$ in ambulatory systolic heart failure patients depending upon the presence of precapillary component as defined by PVR more than 3 WU. Mortality was higher in patients with precapillary component compared to patients with IpcPH and no PH. PVR $>3.5 \mathrm{WU}$ and pulmonary artery compliance (PAC) $<2 \mathrm{~mL} / \mathrm{mmHg}$ identified patients with highest mortality (23). Systolic heart failure patients with vasodilator response to nitroprusside defined by a decrease in PVR <2.5 WU without decrease in systolic blood pressure had a 3 -month mortality of $3.8 \%$ compared to $40 \%$ without vasodilator response (27). The development of RHF due to increased afterload imparted by pulmonary circulation portends poor prognosis in patients with $\mathrm{HFrEF}$ (3).

\section{Pathophysiology of PH in HFrEF}

The pathophysiology of $\mathrm{PH}$ due to HFrEF involves a 
series of changes in pulmonary circulation driven by chronic left atrial hypertension. In the initial stages of systolic heart failure, elevated left-sided filling pressures in response to exercise or volume overload may cause a transient and passive backward transmission of pressures to the pulmonary circuit with an increase in PA pressures and without significant increase in PVR (28). The left atrium serves a protective role, preventing excessive transmission of elevated ventricular filling pressures and volume to the pulmonary circulation. With the progression of left ventricular systolic dysfunction (LVSD), left atrial remodeling occurs with increased left atrial size and stiffness, decreased contractility, and persistent left atrial hypertension. Atrial arrhythmias that develop as a result of elevated left atrial pressure and left atrial remodeling exacerbate $\mathrm{PH}(7)$.

Acute rise in pulmonary venous pressure due to LVSD can stress the pulmonary capillary circulation, breaching the alveolar-capillary unit with resultant cardiogenic pulmonary edema which may be reversible (29). The alveolar-capillary stress fracture coined by West et al. due to acute rise in pulmonary venous pressures can invoke the release of growth factors, neurohormones, cytokines that promote vascular remodeling in the long-term (30). Also, chronically elevated left atrial pressures result in pulmonary capillary and arteriolar remodeling with intimal fibrosis, medial hypertrophy, and vascular smooth muscle proliferation. With progression in pulmonary vascular remodeling, endothelial dysfunction and pulmonary vasoconstriction occurs with an increase in PVR and a decrease in PAC (7). Hypoxia due to impaired gas exchange from alveolar-capillary remodeling can result in pulmonary vasoconstriction and an increase in PVR.

Pulmonary vascular endothelial dysfunction ensues as a result of pulmonary vascular injury and remodeling from chronically elevated left-sided filling pressures. The pulmonary vascular endothelium plays a crucial role in maintaining pulmonary vascular tone by releasing vasodilator and vasoconstrictor mediators. Nitric oxide (NO) pathway, endothelin system, prostacyclin $\left(\mathrm{PGI}_{2}\right)$, and endothelium-derived hyperpolarizing factor are important pathways and mediators that regulate pulmonary vascular tone. Studies in patients with systolic heart failure have shown decreased NO bioavailability as a cause for $\mathrm{PH}$ $(31,32)$. Decreased substrate availability for NO production (L-arginine) and/or dysregulated arginine metabolism with an accumulation of methylated arginine metabolites that inhibit endothelial nitric oxide synthase (eNOS) may be responsible for decreased NO production in congestive heart failure (33). Endothelin-1, a potent vasoconstrictor of the systemic and pulmonary circulation, is elevated in patients with chronic systolic failure $(34,35)$. Elevated plasma endothelin 1 (ET-1) levels correlated with the severity of systolic heart failure and portend poor survival (35). Plasma ET-1 concentrations in the pulmonary circulation positively correlated with PVR in patients with systolic heart failure signifying the role of vasoconstrictor peptide in the pathogenesis of $\mathrm{PH}$ in systolic heart failure (36). Tumor necrosis factor-alpha, elevated in chronic systolic heart failure, has been shown to increase plasma ET-1 concentrations $(37,38)$. Also, the downregulation of endothelin B receptors (promotes clearance of ET-1) and upregulation of endothelin A receptors tip the balance towards pulmonary vasoconstriction (39). ET-1 increases the production of growth factors and thereby promotes adverse pulmonary vascular remodeling by increasing smooth muscle proliferation and collagen deposition (40). The natriuretic peptide (NP) system in chronic systolic heart failure plays an important role in opposing the maladaptive compensatory mechanisms of the sympathetic nervous system and Renin-Angiotensin systems activated in chronic systolic heart failure. NP has vasodilatory, natriuretic, and antiproliferative actions. Despite the increase in NP levels in chronic systolic heart failure, the response is blunted due to decreased levels of active brain natriuretic peptide (BNP) fragments from increased clearance and enzymatic degradation, increased levels of ET-1, decreased NP receptors (NPR) and desensitization (41). Animal models have shown the vasodilatory effects of NP in the pulmonary circulation and the lack of NPR-A in the pathogenesis of PH. Natriuretic peptides also exhibit smooth muscle antiproliferative properties, thereby preventing adverse pulmonary vascular remodeling. The antiproliferative effects of NPR-C signaling to prevent adverse pulmonary vascular remodeling has also been demonstrated in animal studies (42). Furthermore, NO-soluble guanylate cyclase and NP-particulate guanylate cyclase signaling act in concert to maintain vascular tone.

\section{Right ventricle failure in HFrEF}

The RV is a thin-walled, crescent-shaped chamber that is embryologically different compared to the left ventricle (LV) and can adapt to volume overload better than pressure load. The energy expenditure of RV to maintain cardiac output is one-fifth of the LV because pulmonary vasculature 
is a highly compliant and low-pressure circuit (43). RV contractility is maintained through contraction of the free wall, $L V$, and interventricular septum. $L V$ contraction contributes to $20-40 \%$ of the RV contractility, and the interventricular septum plays an important role in maintaining RV cardiac output, especially when RV free wall may be diseased $(44,45)$. RV systolic function in HFrEF may be significantly impaired with decreased LV contractility and further impairs LV preload.

The RV is often the ignored ventricle in prognostication of ischemic and nonischemic dilated cardiomyopathies. RVD in dilated or ischemic cardiomyopathies may occur due to increased afterload to the RV or primary myopathic process affecting the RV or combination of both. In nonischemic dilated cardiomyopathies, the primary myopathic process can affect both ventricles. Genetic and acquired cardiomyopathies like arrhythmogenic cardiomyopathy (AC) and sarcoidosis, respectively may have isolated RV or Biventricular involvement with predominant RVD (46). With the progression of chronic systolic heart failure, RVD may predominate with resultant irreversible liver and renal dysfunction (47). Assessment of RV systolic and diastolic function and RV afterload is fraught with limitations of echocardiographic assessment due to RV anatomy, interobserver variability, snapshot assessment of hemodynamics by right heart catheterization, lack of widespread use of cardiac magnetic resonance imaging and uniform definition of RHF.

$\mathrm{RV}$ function as assessed by right ventricular ejection fraction (RVEF) at rest and exercise is an independent predictor of survival, functional capacity, and need for inotropes or mechanical circulatory support (48-50). In a cohort of 423 patients with HFrEF and no coronary artery disease, Pueschner et al. identified RVD as defined by RVEF $<35 \%$ as an independent predictor of cardiac mortality. The study identified left ventricular ejection fraction (LVEF) and transpulmonary gradient (presence of $\mathrm{CpcPH}$ ) as independent predictors of RVD (51).

The development and severity of RVD with $\mathrm{PH}-$ HFrEF vary in each patient depending on the degree of involvement of RV by primary process, neurohormonal activation, and severity of $\mathrm{PH}$ and remodeling of the pulmonary vasculature. The afterload sensitivity of $\mathrm{RV}$ makes it susceptible to adverse remodeling with chronically elevated PA pressures. Chronically elevated RV afterload results in RV chamber dilation, functional tricuspid regurgitation, impaired $\mathrm{LV}$ filling due to interventricular dependence, and pericardial constraint (52). Impairment of $\mathrm{RV}$ diastolic function due to maladaptive remodeling of RV has been reported in patients with HFrEF and could be a result of increased RV afterload and primary myopathic process affecting RV (53).

\section{Right ventricle-pulmonary artery interaction in HFrEF}

The development and progression of pulmonary vascular disease and development of RVF in left-sided heart disease require an understanding of the components of $\mathrm{RV}$ afterload i.e., pulmonary artery compliance (PAC), resistance, and characteristic impedance. PAC, a measure of elasticity and vessel size, is distributed throughout the entire pulmonary arterial tree with proximal arteries contributing only $20 \%$ of the total arterial compliance (54). The resistance in the pulmonary circulation is predominantly in the distal pulmonary vascular bed (55). The hemodynamic definitions for classification of $\mathrm{PH}$ and prognostication incorporate only the resistive load (PVR) which may not truly represent the total RV afterload.

PAC and PVR are inversely related with hyperbolic relationship i.e., in initial stages, a small increase in PVR result in a large decrease in compliance. As systolic heart failure progresses, increase in resistance results in small changes in compliance due to maximal arterial stiffness (56). This resistance-compliance curve ( $\mathrm{R}-\mathrm{C})$ in the absence of left-sided heart disease is constant (55). In HFrEF, there is an increase in impedance to the pulmonary circulation indicative of a pulsatile load with a shift in $\mathrm{R}-\mathrm{C}$ curve to the left i.e., larger decrease in compliance for a given resistance and can be altered with changes in PCWP and vasodilator therapy $(55,57)$. The increase in PCWP results in high amplitude reflected waves arriving early before RV ejection is complete, thereby decreasing PAC and increasing the RV pulsatile load (58). In patients with ESHF, low PAC $<2.5 \mathrm{~mL} / \mathrm{mmHg}$ (pulsatile load) was predictive of RVD and mortality compared to PVR (59). Effective arterial elastance (Ea) $>1.03 \mathrm{mmHg} / \mathrm{mL}$, a hemodynamic measure of total RV afterload (pulsatile and resistive components) was predictive of severe RVD and mortality in patients with HFrEF and normal PVR. Diastolic pulmonary gradient (DPG) and combination of elevated DPG $>7 \mathrm{mmHg}$ and PVR $>3 \mathrm{WU}$ did not predict mortality (60).

It is important to highlight that the response of the RV to the pulsatile afterload determines risk of RVF and shortterm survival in patients undergoing cfLVAD implantation. Results of a single-center retrospective study investigating 
the pulsatile load to RV in patients undergoing cfVLAD implantation showed that patients with a combination of low PAC indexed to body surface area (PACi) and elevated CVP: PCWP ratio predicted the highest risk of early RHF (64\%) and 6-month mortality (61). This study underscores the importance of incorporation of RV pulsatile load assessment coupled with parameters of RV systolic function for RHF risk prediction and mitigating RV failure.

\section{Fate of Pulmonary hypertension after cfLVAD}

cFLVAD implantation is used as a bridge to heart transplantation in patients with ESHF and $\mathrm{PH}$ refractory to medical therapy. Single-center studies have shown marked improvement and normalization of PA pressures after cfLVAD implantation, thereby attaining the goal of transplant eligibility $(15,16,18,62)$. RV afterload reduction with normalization of $\mathrm{PA}$ pressures due to $\mathrm{LV}$ mechanical unloading may improve RV-PA coupling and prevent late RVD. There is significant heterogeneity among studies that report a decrease in PA pressures and PVR after LVAD in regards to device used (pulsatile versus continuous flow), severity of $\mathrm{PH}$, differentiation between CpcPH and IpcPH, lack of pulmonary vasodilator data and the etiology for persistent $\mathrm{PH}$. The time course for improvement in PA pressures and PVR after cfLVAD implantation ranges from one week to maximal benefit seen at 6months $(15,16,18,63-66)$. Persistence of $\mathrm{PH}$ after cfLVAD could be due to ineffective unloading, persistent MR, or the presence of comorbid conditions like obstructive sleep apnea or chronic obstructive pulmonary disease. Mechanical unloading to decrease moderate-severe MR may be challenging in patients with severe RVD and can contribute to persistent PH (67). Studies have shown similar post-transplant survival in patients with elevated PVR bridged with LVAD compared to patients with normal PVR $(68,69)$. However, one single-center retrospective study by Tsukashita $e t$ al. showed that patients with elevated PVR $>5 \mathrm{WU}$ before cfLVAD implantation had higher in-hospital mortality and primary graft failure after HT despite normalization of transpulmonary gradient (TPG) and PVR after cfLVAD. Pulmonary vasodilator use in the elevated PVR group in this study was only $40 \%$ (65). An explanation for this finding that is not explored and may be challenging to prove is that the histological changes in the pulmonary vascular bed may not be reversible despite normalization of PVR. As a result, $\mathrm{PH}$ may be unmasked with the perioperative insults to pulmonary vasculature from mechanical ventilation, hypoxia and hypercarbia, systemic inflammatory response with release of cytokines, and pulmonary vasoconstrictors like endothelin-1 during cardiopulmonary bypass, anesthesia, protamine administration and fluid shifts (70). There is evidence that DPG $>5 \mathrm{mmHg}$ at baseline cfLVAD speed and difference in DPG gradient $>3 \mathrm{mmHg}$ between set speed and highest speed during a ramp protocol may be indicative of persistent pre-capillary $\mathrm{PH}$ with associated increased HF hospitalizations and decreased one-year survival $(71,72)$.

\section{RHF post cfLVAD implantation}

The management of $\mathrm{PH}$ after cfLVAD implantation is paramount, as sustained elevated filling pressures can precipitate RHF. RHF can occur in up to $10-30 \%$ of patients after cFLVAD implantation. The incidence varies due to heterogeneity in definitions used to define RHF (73-75). The Interagency Registry for Mechanical Circulatory Support (INTERMACS) definition of RVF after cfLVAD implantation is based on clinical symptoms, physical examination, end-organ function, hemodynamics assessment and RVF severity based on the use of mechanical support or duration of inotrope use. Prolonged use of inotropes (>14 days) and use of right ventricular assist device were identified as the highest risk cohort for increased mortality at 2 years (76). RHF post cfLVAD implantation is associated with decreased survival, increased length of stay, and increased risk of gastrointestinal bleeding $(77,78)$. Early RHF occurs immediately post implantation and is attributed to (I) increase in RV preload due to increase in cardiac output contributed by the left ventricular assist device and volume resuscitation during surgery (II) Interventricular septal shift to the left due to LV unloading with loss of septal contribution to RV systolic function (III) increased afterload due to pre-capillary component $\mathrm{PH}$, hypoxia or hypercarbia or suboptimal unloading (79) (Figure 1). The etiology of late RHF is less defined, as it is thought to be in part due to myriad of etiologies. Occurring weeks to months after cfLVAD implantation in $10 \%$ of patients, late RHF is associated with decreased survival, quality of life, multiorgan dysfunction and decreased post-heart transplant survival (80-82). Late RHF may be due to inefficient left ventricular unloading in the setting of moderate-severe RVD and persistent precapillary pulmonary hypertension all of which increase right ventricular overload (83). Thus, a strategy to maintain a low right ventricular afterload in the 


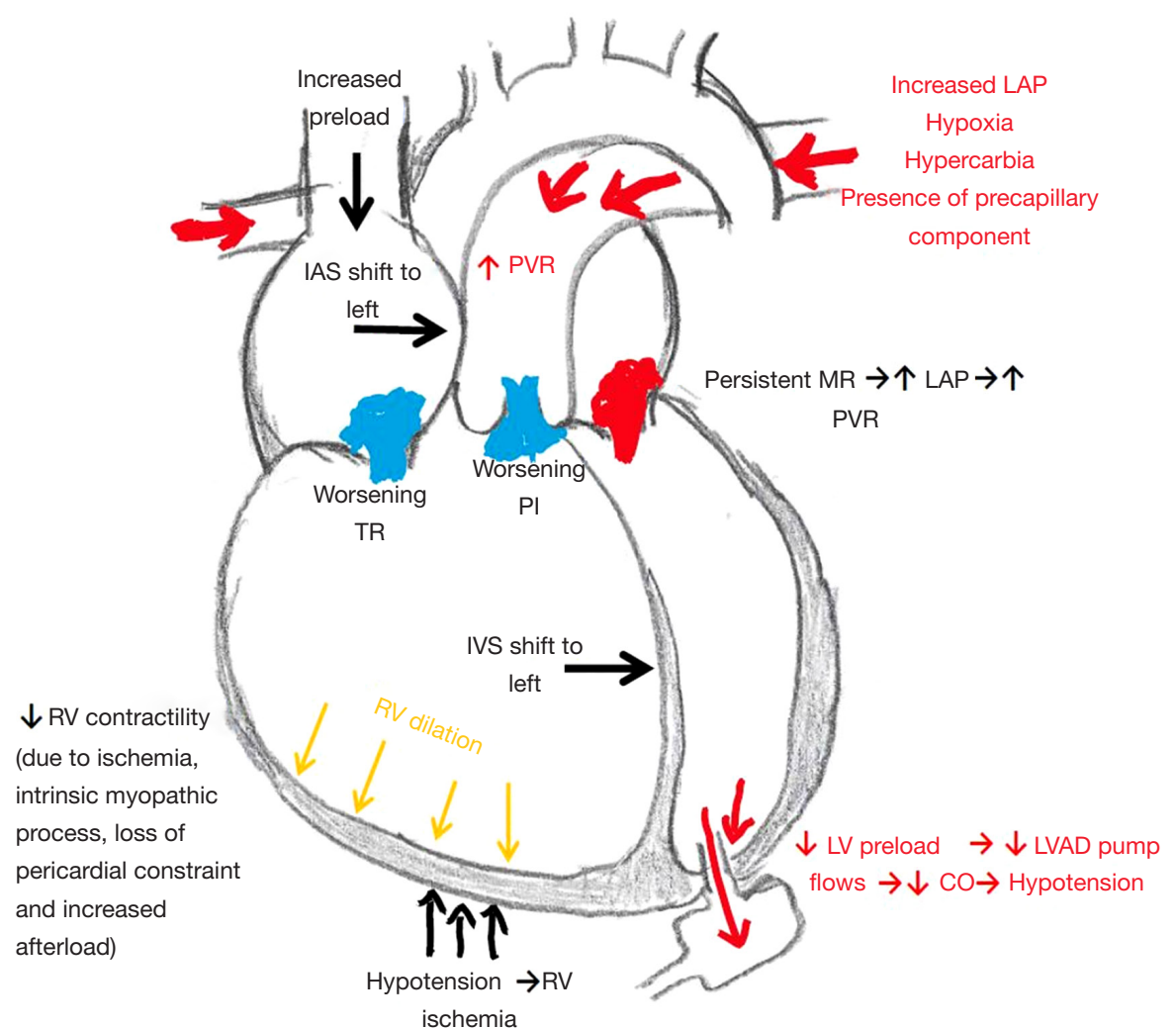

Figure 1 Pathophysiology of Right ventricular failure after continuous flow left ventricular assist device implantation. IVS, interventricular septum; TR, tricuspid regurgitation; MR, mitral regurgitation; LAP, left atrial pressure; PI, pulmonic insufficiency; IAS, interatrial septum; $\mathrm{RV}$, right ventricle; $\mathrm{LV}$, left ventricle; LVAD, left ventricular assist device; $\mathrm{CO}$, cardiac output.

immediate post-operative period and long-term is key to improve clinical outcomes.

\section{Hemodynamic assessment after cfLVAD}

There is discordance between clinical assessment of volume status and invasive hemodynamic assessment after cfLVAD implantation. Invasive hemodynamic studies by Imamura et al. have shown that only $50 \%$ of patients have optimal right and left-sided filling pressures defined as CVP $<12 \mathrm{mmHg}$ and PCWP $<18 \mathrm{mmHg}$, respectively after a median duration of 236 days post cfLVAD implantation. After hemodynamic optimization, only $61 \%$ achieved the optimal right and left-sided filling pressures and fick cardiac index $(\mathrm{CI})>2.2 \mathrm{~L} / \mathrm{min} / \mathrm{m}^{2}$ (84). Results of a singlecenter retrospective study by Shah et al. showed that $29 \%$ of patients had elevated right and left-sided filling pressures and low CI at 3 months after cfLVAD implantation (85). The hemodynamic optimization with ramp protocol performed by Imamura et al. and Shah et al. showed that
CVP remained elevated despite a decrease in PCWP and an increase in CI suggestive of persistent RV dysfunction $(84,85)$. Also, a low PA pulsatility index at baseline speed was a predictor of failure to achieve optimal hemodynamics. In other words, underlying RV systolic dysfunction may limit the pump speed adjustments to optimize left-sided filling pressures (84). However, lack of inclusion of PA pressures, PVR, and TPG in these studies do not allow us to conclude if elevated CVP was due to impaired intrinsic RV contractility or increased RV afterload or both.

American Association for Thoracic Surgery/The International Society of Heart and Lung transplantation guidelines recommend pump speed adjustments in the immediate post-operative period and during chronic support to allow maximal unloading using transthoracic echocardiogram and is consensus-based. The recommendations are to adjust pump speed to prevent excessive interventricular septal shift to the left, decrease MR and allow intermittent aortic valve opening (86). No formal guidelines regarding hemodynamic goals and 
optimization of pulmonary vascular disease exist. There is variability among centers to evaluate optimal cFLVAD support periodically using echocardiography and invasive hemodynamic monitoring.

\section{Principles of cfLVAD management}

Management of patients implanted with cfLVAD should be individualized and tailored with a combination of optimal mechanical unloading and medical therapies. The goals of cFLVAD optimization include (I) decrease left-sided filling pressures with resultant reduction in pulmonary venous pressures and right ventricular afterload (II) optimize cFLVAD pump speed to prevent excessive interventricular septal shift and worsen RHF (III) sinus rhythm restoration in patients with atrial fibrillation (IV) management of ventricular arrhythmias (V) optimize medical management to decrease preload with diuretics, precapillary component of $\mathrm{PH}$ with pulmonary vasodilators and systemic blood pressure with antihypertensive agents (VI) management of comorbidities like obstructive sleep apnea and chronic obstructive pulmonary disease to decrease the precapillary component of $\mathrm{PH}$. Hemodynamic optimization in the perioperative and postoperative period is crucial to prevent prolonged mechanical ventilation and RHF. Periodic optimization of cfLVAD patients should include echocardiographic and invasive hemodynamic assessment.

\section{Pulmonary vasodilator therapy after cfLVAD implantation}

Management of $\mathrm{PH}$ in patients with cfLVAD can be divided into two phases to prevent early and late RHF due to uncorrected $\mathrm{PH}$ and increase the chance for transplant eligibility: perioperative period (preoperative, intraoperative, postoperative) and chronic support (>30 days) after implant.

\section{Preoperative period}

Unloading the LV using percutaneous mechanical support (pMCS) and systemic vasodilators decreases pulsatile load to RV by decreasing left-sided filling pressures. The use of pulmonary vasodilators like nitric oxide or PDE5i preoperatively to decrease resistive load is not currently supported by robust data. Pulmonary vasodilator use before cfLVAD implantation in a retrospective single-center study including 16 patients and a case report prevented
RHF $(87,88)$. INTERMACS analysis of PDE-5i use prior to cfLVAD implantation resulted in a $31 \%$ higher risk of RHF requiring prolonged inotropic support more than 14 days (89). Several limitations of this report preclude us from drawing conclusions (I) Lack of data in regards to the proportion of patients with $\mathrm{CpcPH}$ and $\mathrm{IpcPH}$ and (II) Lack of data regarding preimplant use of pMCS for unloading to protect excessive preload to $\mathrm{LV}$ due to pulmonary vasodilator use.

In the immediate postoperative setting, preservation of $\mathrm{RV}$ function is achieved primarily through optimizing RV preload, RV contractility, and RV afterload (79). Pulmonary vasodilators are used during the immediate postoperative period to decrease RV afterload. Inhaled nitric oxide (iNO), a selective pulmonary vasodilator is commonly used in the intraoperative and postoperative period to reduce PVR. iNO, delivered through the mechanical ventilation circuit or supplemental high flow oxygen, increases the production of cyclic guanosine monophosphate (cGMP) by binding to enzyme guanylate cyclase in vascular smooth muscle and causes pulmonary vascular smooth muscle vasodilation. iNO also improves ventilation-perfusion matching, thereby decreasing hypoxic pulmonary vasoconstriction and PVR (90). Single-center non-randomized clinical studies using iNO after cfLVAD implantation have shown improvement in PVR, PA pressures, CVP, CI, and cfLVAD flows $(91,92)$. Two randomized clinical trials using iNO during the perioperative period yielded conflicting results. In a multicenter randomized trial designed by Patapov et al., iNO initiated at 40 parts per million (ppm) before weaning cardiopulmonary bypass $(\mathrm{CPB})$ and continued for 48 hours did not significantly decrease the incidence of RHF, duration of mechanical ventilation, length of stay and survival. However, several limitations of the study including crossover of patients to open-label iNO, variability in patient susceptibility to RHF, intraoperative factors and finally, individual center management in the post-operative setting precludes us from drawing conclusions regarding iNO use in the perioperative period (93). Another, small single-center study that randomized patients with high PVR after weaning CPB to iNO or placebo showed improvement in cLVAD flows and decrease in PA pressures in the iNO group (94). With limited evidence and challenges designing a randomized trial due to described limitations above, iNO use can be recommended for patients at high risk for postoperative RVF, hemodynamic criteria for $\mathrm{CpcPH}$ or elevated PVR after weaning CPB. Timing of iNO administration empirically after induction 
of anesthesia versus after weaning $\mathrm{CPB}$ varies widely across centers and has not been studied. Effects of iNO may be potentiated with addition of PDE5 inhibition by preventing breakdown of cGMP. Due to increased cost and risk of methemoglobinemia, iNO weaning may be bridged with the use of oral pulmonary vasodilators like PDE5i (95) or alternative use of inhaled prostacyclins should be considered. Inhaled epoprostenol, a prostacyclin analog was reported to lower mean PA pressures after cfLVAD implantation but resulted in increased post-operative bleeding likely due to impaired platelet aggregation (96). Inhaled iloprost, a prostacyclin analog with a half-life of 20-30 minutes and intermittent dosing has been studied in combination with iNO in cfLVAD patients in the postoperative period and has a synergistic effect in improving PVR and cfLVAD flows. Inhaled iloprost use may allow for weaning of iNO without rebound increase in PA pressures (97).

Milrinone, a phosphodiesterase type 3 inhibitor has positive inotropic and pulmonary vasodilatory properties by increasing cAMP and cGMP in cardiac myocytes and vascular smooth muscle, respectively $(98,99)$. The combination of positive inotropic and pulmonary vasodilator effects makes intravenous (IV) milrinone a desirable inotrope for postoperative RHF (100). However, the systemic vasodilatory effects of IV milrinone can be deleterious for $\mathrm{RV}$ function by potentiating $\mathrm{RV}$ ischemia. Hence, milrinone in combination with a vasopressor with less pulmonary vasoconstrictive effect (vasopressin) may help mitigate the hypotensive side effect (101). Inhaled milrinone for 24 hours after cfLVAD implant decreased PA pressures without systemic vasodilatory side effects but there was a $30 \%$ incidence of ventricular arrhythmias (102). Inhaled milrinone is not widely used and more data regarding safety and efficacy needs to be available before routine use.

The data to support long-term use of pulmonary vasodilator therapy post cfLVAD implant is not robust, with most data evaluating the use of PDE5i in this patient population. The current International Society of Heart and Lung Transplant guidelines recommend only the selective use of PDE5i for the management of RVD in the setting of $\mathrm{PH}$ and the evidence regarding efficacy is not strong and based on consensus opinion (Class IIb; level of evidence C) (103). Several questions regarding the use of endothelin receptor antagonists (ERA), soluble guanylate cyclase stimulators and prostacyclin analogs, hemodynamic criteria, and duration of use in cfLVAD patients remain unanswered.

PDE5i prevents the degradation of cGMP by inhibiting PDE5 and increases nitric oxide mediated pulmonary vasodilation (104). In addition to the pulmonary vasodilatory effects, there is animal data to suggest the positive inotropic effect of sildenafil (PDE5i) by indirectly increasing cAMP due to inhibition of cGMP sensitive PDE3 (105). Sildenafil is the most commonly used PDE5i in clinical practice and trials of cfLVAD patients. Tedford et al. conducted the largest clinical study of PDE5i in cfLVAD patients. The study, performed as an open-label clinical trial, identified 58 of 138 patients with a persistent $\mathrm{PVR}>3 \mathrm{WU} 1$ to 2 weeks after cfLVAD implantation despite a decrease in left-sided filling pressures (PCWP $<15 \mathrm{mmHg}$ ). Of these 58 patients, 26 patients were started on sildenafil at a dose of $25 \mathrm{mg} 3$ times daily with a target dose of $75 \mathrm{mg} 3$ times daily that was achieved within one week of initiation. There was a significant reduction in the mPAP from 36.5 to $24.3 \mathrm{mmHg}$ and PVR from 5.87 to $2.96 \mathrm{WU}$ after two to four weeks of sildenafil as compared to the subgroup that was not initiated on pulmonary vasodilator therapy. The improvement in PVR was maintained at 15 weeks after the initiation of sildenafil. In addition to improvement in PA pressures, the patients in the sildenafil group demonstrated a significant improvement in RV contractility as measured by their RV contractility index (106). A single-center retrospective study analyzed the data of advanced heart failure patients who underwent cfLVAD implantation with pre-existing preoperative $\mathrm{PH}$ and RVD as defined by unfavorable RV geometry, reduced tricuspid annular plane systolic excursion (TAPSE) $<15 \mathrm{~mm}$, and tissue Doppler $\mathrm{S}$ wave velocities less than 10 centimeters/second at the tricuspid annulus. The patients were divided into 2 groups based on sildenafil administration. Sildenafil administered with a mean daily dose of $56.2 \mathrm{mg}$ significantly reduced PVR, TPG (2.6 WU vs. $3.05 \mathrm{WU} ; 11.7$ vs. $14.8 \mathrm{mmHg}$ respectively) and increased CI (2.8 versus $\left.2.05 \mathrm{~L} / \mathrm{min} / \mathrm{m}^{2}\right)$ compared to the group not receiving sildenafil. The incidence of RHF was significantly higher in the group not receiving sildenafil establishing the potential for PDE5i use in post cfLVAD patients (87). A retrospective single-center study evaluated 318 cfLVAD patients and found no difference in 30-day readmission, inotrope or epoprostenol duration, survival, duration of mechanical ventilation, length of stay, and improvement in RVD in patients taking sildenafil. Lack of hemodynamic and echocardiographic data to assess the degree of $\mathrm{PH}$, presence of precapillary component and RV function pre and postoperatively is a major limitation of this study (107). Besides improvement in PA pressures and RV function with sildenafil, a potential benefit of reduction in ischemic stroke and pump thrombosis has been reported in 
a single-center study of patients supported on Heartmate II (108). The benefit may be due to PDE5 inhibition of platelet aggregation. There was no evidence of increased gastrointestinal bleeding in cFLVAD patients on sildenafil in a single-center study despite the inhibition of platelet aggregation (109).

Though the majority of data has primarily focused on the use of PED5i to manage post cfLVAD PH, there has been growing interest in the investigation of ERA for the management of $\mathrm{PH}$ post cFLVAD implantation. A singlecenter retrospective study by LaRue et al. evaluated the use of bosentan for management of secondary $\mathrm{PH}$ and demonstrated a decrease in echocardiographic estimated PVR from $3.93 \pm 1.53 \mathrm{WU}$ in the preoperative setting to $2.58 \pm 1.05$ WU 3 to 6 months post cFLVAD. There was no placebo group and incidence of RHF was $18 \%$. Bosentan was initiated a median 37 days after cfLVAD implantation. Liver function abnormalities were seen in $6 \%$ of patients and normalized after drug discontinuation (110). The safety profile of ERA in cfLVAD patients needs to be investigated further. The SOPRANO (Study of Macitentan in Patients with Pulmonary Hypertension Post-Left Ventricular Assist Device Implantation) trial, the first multi-center randomized placebo-controlled trial to evaluate safety and efficacy of pulmonary vasodilator (macitentan) in patients with precapillary $\mathrm{PH}$ post cfLVAD, completed enrollment and pending results (ClinicalTrials.gov Identifier: NCT02554903).

\section{Future directions}

With the improvement in cfLVAD device technology, shifting focus to stratifying individual patient risk for complications after cFLVAD implantation may help improve outcomes. There is a huge need for multi-center randomized clinical trials of pulmonary vasodilator use in predefined subset of patients with $\mathrm{PH}$ post cfLVAD implantation along with serum biomarkers to assess the degree of improvement of pulmonary vascular hemodynamics, pulmonary vascular remodeling and clinical outcomes. Indeed the results of the randomized clinical trial of macitentan targeting a reduction in PVR after cfLVAD will provide the first evidence to date that this strategy of applying pulmonary vasodilatory therapy that is used for WHO Group I PH can have an impact in patients with an elevated PVR post cfLVAD. The use of pulmonary vasodilators prior to cfLVAD implantation needs to be clearly defined and studied before drawing conclusions about the negative impact.

\section{Conclusions}

Persistent $\mathrm{PH}$ after cfLVAD implantation can lead to RHF. Ineffective mechanical unloading in the setting of moderatesevere RVD, persistent MR despite maximal unloading and precapillary component of $\mathrm{PH}$ are contributing factors to persistent $\mathrm{PH}$ after cfLVAD implantation. Pulmonary vasodilator use in cfLVAD population lacks robust data. SOPRANO, a multicenter center randomized clinical trial will provide input regarding benefits and indications for use in the cfLVAD population.

\section{Acknowledgments}

Funding: None.

\section{Footnote}

Provenance and Peer Review: This article was commissioned by the Guest Editors (Dr. Debabrata Mukherjee and Dr. Jose B. Cruz Rodriguez) for the series "Heart Failure Update and Advances in 2021" published in Annals of Translational Medicine. The article was sent for external peer review organized by the Guest Editors and the editorial office.

Peer Review File: Available at http://dx.doi.org/10.21037/ atm-20-4710

Conflicts of Interest: All authors have completed the ICMJE uniform disclosure form (available at http://dx.doi. org/10.21037/atm-20-4710). The series "Heart Failure Update and Advances in 2021" was commissioned by the editorial office without any funding or sponsorship. Dr. Rame reports that he is in Actelion Pharmaceuticals (now Jansen Inc): Steering Committee Member for the SoPrANO Trial investigating Macitentan in patients with Pulmonary Hypertension after Left Ventricular Assist Device. The authors have no other conflicts of interest to declare.

Ethical Statement: The authors are accountable for all aspects of the work in ensuring that questions related to the accuracy or integrity of any part of the work are appropriately investigated and resolved. 
Open Access Statement: This is an Open Access article distributed in accordance with the Creative Commons Attribution-NonCommercial-NoDerivs 4.0 International License (CC BY-NC-ND 4.0), which permits the noncommercial replication and distribution of the article with the strict proviso that no changes or edits are made and the original work is properly cited (including links to both the formal publication through the relevant DOI and the license). See: https://creativecommons.org/licenses/by-nc-nd/4.0/.

\section{References}

1. Guazzi M, Borlaug BA. Pulmonary hypertension due to left heart disease. Circulation 2012;126:975-90.

2. Rosenkranz S, Gibbs JS, Wachter R, et al. Left ventricular heart failure and pulmonary hypertension. Eur Heart J 2016;37:942-54.

3. Ghio S, Gavazzi A, Campana C, et al. Independent and additive prognostic value of right ventricular systolic function and pulmonary artery pressure in patients with chronic heart failure. J Am Coll Cardiol 2001;37:183-8.

4. Khan Z, Gholkar G, Tolia S, et al. Effect of sacubitril/ valsartan on cardiac filling pressures in patients with left ventricular systolic dysfunction. Int J Cardiol 2018;271:169-73.

5. Asgar AW, Mack MJ, Stone GW. Secondary mitral regurgitation in heart failure: pathophysiology, prognosis, and therapeutic considerations. J Am Coll Cardiol 2015;65:1231-48.

6. Aras MA, Psotka MA, De Marco T. Pulmonary Hypertension Due to Left Heart Disease: an Update. Curr Cardiol Rep 2019;21:62.

7. Al-Omary MS, Sugito S, Boyle AJ, et al. Pulmonary Hypertension Due to Left Heart Disease: Diagnosis, Pathophysiology, and Therapy. Hypertension 2020;75:1397-408.

8. Lewis GD, Shah R, Shahzad K, et al. Sildenafil improves exercise capacity and quality of life in patients with systolic heart failure and secondary pulmonary hypertension. Circulation 2007;116:1555-62.

9. Guazzi M, Samaja M, Arena R, et al. Long-term use of sildenafil in the therapeutic management of heart failure. $\mathrm{J}$ Am Coll Cardiol 2007;50:2136-44.

10. Guazzi M, Vicenzi M, Arena R, et al. PDE5 inhibition with sildenafil improves left ventricular diastolic function, cardiac geometry, and clinical status in patients with stable systolic heart failure: results of a 1-year, prospective, randomized, placebo-controlled study. Circ Heart Fail
2011;4:8-17.

11. Guazzi M, Vicenzi M, Arena R. Phosphodiesterase 5 inhibition with sildenafil reverses exercise oscillatory breathing in chronic heart failure: a long-term cardiopulmonary exercise testing placebo-controlled study. Eur J Heart Fail 2012;14:82-90.

12. Kim KH, Kim HK, Hwang IC, et al. PDE 5 inhibition with udenafil improves left ventricular systolic/diastolic functions and exercise capacity in patients with chronic heart failure with reduced ejection fraction; A 12-week, randomized, double-blind, placebo-controlled trial. Am Heart J 2015;169:813-22.e3.

13. Ozturk P, Engin AY, Nalbantgil S, et al. Comparison of continuous-flow and pulsatile-flow blood pumps on reducing pulmonary artery pressure in patients with fixed pulmonary hypertension. Artif Organs 2013;37:763-7.

14. Martin J, Siegenthaler MP, Friesewinkel O, et al. Implantable left ventricular assist device for treatment of pulmonary hypertension in candidates for orthotopic heart transplantation-a preliminary study. Eur J Cardiothorac Surg 2004;25:971-7.

15. Kutty RS, Parameshwar J, Lewis C, et al. Use of centrifugal left ventricular assist device as a bridge to candidacy in severe heart failure with secondary pulmonary hypertension. Eur J Cardiothorac Surg 2013;43:1237-42.

16. Mikus E, Stepanenko A, Krabatsch T, et al. Reversibility of fixed pulmonary hypertension in left ventricular assist device support recipients. Eur J Cardiothorac Surg 2011;40:971-7.

17. Zimpfer D, Zrunek P, Sandner S, et al. Post-transplant survival after lowering fixed pulmonary hypertension using left ventricular assist devices. Eur J Cardiothorac Surg 2007;31:698-702.

18. Salzberg SP, Lachat ML, von Harbou K, et al. Normalization of high pulmonary vascular resistance with LVAD support in heart transplantation candidates. Eur J Cardiothorac Surg 2005;27:222-5.

19. Nair PK, Kormos RL, Teuteberg JJ, et al. Pulsatile left ventricular assist device support as a bridge to decision in patients with end-stage heart failure complicated by pulmonary hypertension. J Heart Lung Transplant 2010;29:201-8.

20. Mehra MR, Canter CE, Hannan MM, et al. The 2016 International Society for Heart Lung Transplantation listing criteria for heart transplantation: A 10-year update. J Heart Lung Transplant 2016;35:1-23.

21. Wood P. Pulmonary hypertension with special reference to the vasoconstrictive factor. Br Heart J 1958;20:557-70. 
22. Simonneau G, Montani D, Celermajer DS, et al. Haemodynamic definitions and updated clinical classification of pulmonary hypertension. Eur Respir J 2019;53:18019153.

23. Miller WL, Grill DE, Borlaug BA. Clinical features, hemodynamics, and outcomes of pulmonary hypertension due to chronic heart failure with reduced ejection fraction: pulmonary hypertension and heart failure. JACC Heart Fail 2013;1:290-9.

24. Grigioni F, Potena L, Galie N, et al. Prognostic implications of serial assessments of pulmonary hypertension in severe chronic heart failure. J Heart Lung Transplant 2006;25:1241-6.

25. Khush KK, Tasissa G, Butler J, et al. Effect of pulmonary hypertension on clinical outcomes in advanced heart failure: analysis of the Evaluation Study of Congestive Heart Failure and Pulmonary Artery Catheterization Effectiveness (ESCAPE) database. Am Heart J 2009; 157:1026-34.

26. Enriquez-Sarano M, Rossi A, Seward JB, et al. Determinants of pulmonary hypertension in left ventricular dysfunction. J Am Coll Cardiol 1997;29:153-9.

27. Costard-Jackle A, Fowler MB. Influence of preoperative pulmonary artery pressure on mortality after heart transplantation: testing of potential reversibility of pulmonary hypertension with nitroprusside is useful in defining a high risk group. J Am Coll Cardiol 1992;19:48-54.

28. Guazzi M, Galie N. Pulmonary hypertension in left heart disease. Eur Respir Rev 2012;21:338-46.

29. West JB, Mathieu-Costello O. Vulnerability of pulmonary capillaries in heart disease. Circulation 1995;92:622-31.

30. Guazzi M, Phillips SA, Arena R, et al. Endothelial dysfunction and lung capillary injury in cardiovascular diseases. Prog Cardiovasc Dis 2015;57:454-62.

31. Porter TR, Taylor DO, Cycan A, et al. Endotheliumdependent pulmonary artery responses in chronic heart failure: influence of pulmonary hypertension. J Am Coll Cardiol 1993;22:1418-24.

32. Cooper CJ, Jevnikar FW, Walsh T, et al. The influence of basal nitric oxide activity on pulmonary vascular resistance in patients with congestive heart failure. Am J Cardiol 1998;82:609-14.

33. Shao Z, Wang Z, Shrestha K, et al. Pulmonary hypertension associated with advanced systolic heart failure: dysregulated arginine metabolism and importance of compensatory dimethylarginine dimethylaminohydrolase-1. J Am Coll Cardiol
2012;59:1150-8.

34. Yanagisawa M, Kurihara H, Kimura S, et al. A novel potent vasoconstrictor peptide produced by vascular endothelial cells. Nature 1988;332:411-5.

35. Pacher R, Stanek B, Hulsmann M, et al. Prognostic impact of big endothelin-1 plasma concentrations compared with invasive hemodynamic evaluation in severe heart failure. J Am Coll Cardiol 1996;27:633-41.

36. Tsutamoto T, Wada A, Maeda Y, et al. Relation between endothelin-1 spillover in the lungs and pulmonary vascular resistance in patients with chronic heart failure. J Am Coll Cardiol 1994;23:1427-33.

37. Levine B, Kalman J, Mayer L, et al. Elevated circulating levels of tumor necrosis factor in severe chronic heart failure. N Engl J Med 1990;323:236-41.

38. Klemm P, Warner TD, Hohlfeld T, et al. Endothelin 1 mediates ex vivo coronary vasoconstriction caused by exogenous and endogenous cytokines. Proc Natl Acad Sci U S A 1995;92:2691-5.

39. Zolk O, Quattek J, Sitzler G, et al. Expression of endothelin-1, endothelin-converting enzyme, and endothelin receptors in chronic heart failure. Circulation 1999;99:2118-23.

40. Galie N, Manes A, Branzi A. The endothelin system in pulmonary arterial hypertension. Cardiovasc Res 2004;61:227-37.

41. Diez J. Chronic heart failure as a state of reduced effectiveness of the natriuretic peptide system: implications for therapy. Eur J Heart Fail 2017;19:167-76.

42. Egom EE, Maher V, El Hiani Y. Evolving use of natriuretic peptide receptor type-C as part of strategies for the treatment of pulmonary hypertension due to left ventricle heart failure. Int J Cardiol 2019;281:172-8.

43. Friedberg MK, Redington AN. Right versus left ventricular failure: differences, similarities, and interactions. Circulation 2014;129:1033-44.

44. Santamore WP, Dell'Italia LJ. Ventricular interdependence: significant left ventricular contributions to right ventricular systolic function. Prog Cardiovasc Dis 1998;40:289-308.

45. Buckberg GD, Group R. The ventricular septum: the lion of right ventricular function, and its impact on right ventricular restoration. Eur J Cardiothorac Surg 2006;29 Suppl 1:S272-8.

46. Corrado D, Basso C, Judge DP. Arrhythmogenic Cardiomyopathy. Circ Res 2017;121:784-802.

47. DeFilippis EM, Guazzi M, Colombo PC, et al. A right ventricular state of mind in the progression of heart 
failure with reduced ejection fraction: implications for left ventricular assist device therapy. Heart Fail Rev 2020. [Epub ahead of print].

48. Di Salvo TG, Mathier M, Semigran MJ, et al. Preserved right ventricular ejection fraction predicts exercise capacity and survival in advanced heart failure. J Am Coll Cardiol 1995;25:1143-53.

49. Meluzin J, Spinarova L, Hude P, et al. Combined right ventricular systolic and diastolic dysfunction represents a strong determinant of poor prognosis in patients with symptomatic heart failure. Int J Cardiol 2005;105:164-73.

50. Juilliere Y, Barbier G, Feldmann L, et al. Additional predictive value of both left and right ventricular ejection fractions on long-term survival in idiopathic dilated cardiomyopathy. Eur Heart J 1997;18:276-80.

51. Pueschner A, Chattranukulchai P, Heitner JF, et al. The Prevalence, Correlates, and Impact on Cardiac Mortality of Right Ventricular Dysfunction in Nonischemic Cardiomyopathy. JACC Cardiovasc Imaging 2017;10:1225-36.

52. Haddad F, Doyle R, Murphy DJ, et al. Right ventricular function in cardiovascular disease, part II: pathophysiology, clinical importance, and management of right ventricular failure. Circulation 2008;117:1717-31.

53. Yu CM, Sanderson JE, Chan S, et al. Right ventricular diastolic dysfunction in heart failure. Circulation 1996;93:1509-14.

54. Saouti N, Westerhof N, Helderman F, et al. RC time constant of single lung equals that of both lungs together: a study in chronic thromboembolic pulmonary hypertension. Am J Physiol Heart Circ Physiol 2009;297:H2154-60.

55. Tedford RJ. Determinants of right ventricular afterload (2013 Grover Conference series). Pulm Circ 2014;4:211-9.

56. Reuben SR. Compliance of the human pulmonary arterial system in disease. Circ Res 1971;29:40-50.

57. Tedford RJ, Hassoun PM, Mathai SC, et al. Pulmonary capillary wedge pressure augments right ventricular pulsatile loading. Circulation 2012;125:289-97.

58. Kussmaul WG, 3rd, Altschuler JA, Matthai WH, et al. Right ventricular-vascular interaction in congestive heart failure. Importance of low-frequency impedance. Circulation 1993;88:1010-5.

59. Dupont M, Mullens W, Skouri HN, et al. Prognostic role of pulmonary arterial capacitance in advanced heart failure. Circ Heart Fail 2012;5:778-85.

60. Tampakakis E, Shah SJ, Borlaug BA, et al. Pulmonary Effective Arterial Elastance as a Measure of Right Ventricular Afterload and Its Prognostic Value in
Pulmonary Hypertension Due to Left Heart Disease. Circ Heart Fail 2018;11:e004436.

61. Grandin EW, Zamani P, Mazurek JA, et al. Right ventricular response to pulsatile load is associated with early right heart failure and mortality after left ventricular assist device. J Heart Lung Transplant 2017;36:97-105.

62. Elhenawy AM, Algarni KD, Rodger M, et al. Mechanical circulatory support as a bridge to transplant candidacy. J Card Surg 2011;26:542-7.

63. Pauwaa S, Bhat G, Tatooles AJ, et al. How effective are continuous flow left ventricular assist devices in lowering high pulmonary artery pressures in heart transplant candidates? Cardiol J 2012;19:153-8.

64. Etz CD, Welp HA, Tjan TD, et al. Medically refractory pulmonary hypertension: treatment with nonpulsatile left ventricular assist devices. Ann Thorac Surg 2007;83:1697-705.

65. Tsukashita M, Takayama H, Takeda K, et al. Effect of pulmonary vascular resistance before left ventricular assist device implantation on short- and long-term post-transplant survival. J Thorac Cardiovasc Surg 2015;150:1352-60, 61.e1-2.

66. Zimpfer D, Zrunek P, Roethy W, et al. Left ventricular assist devices decrease fixed pulmonary hypertension in cardiac transplant candidates. J Thorac Cardiovasc Surg 2007;133:689-95.

67. Tang PC, Haft JW, Romano MA, et al. Right ventricular function and residual mitral regurgitation after left ventricular assist device implantation determines the incidence of right heart failure. J Thorac Cardiovasc Surg 2020;159:897-905.e4.

68. Moayedifar R, Zuckermann A, Aliabadi-Zuckermann A, et al. Long-term heart transplant outcomes after lowering fixed pulmonary hypertension using left ventricular assist devices. Eur J Cardiothorac Surg 2018;54:1116-21.

69. Alba AC, Rao V, Ross HJ, et al. Impact of fixed pulmonary hypertension on post-heart transplant outcomes in bridge-to-transplant patients. J Heart Lung Transplant 2010;29:1253-8.

70. Denault A, Deschamps A, Tardif JC, et al. Pulmonary hypertension in cardiac surgery. Curr Cardiol Rev 2010;6:1-14.

71. Imamura T, Chung B, Nguyen A, et al. Decoupling Between Diastolic Pulmonary Artery Pressure and Pulmonary Capillary Wedge Pressure as a Prognostic Factor After Continuous Flow Ventricular Assist Device Implantation. Circ Heart Fail 2017;10:e003882.

72. Imamura T, Kim G, Raikhelkar J, et al. Decoupling 
Between Diastolic Pulmonary Arterial Pressure and Pulmonary Arterial Wedge Pressure at Incremental Left Ventricular Assist Device (LVAD) Speeds Is Associated With Worse Prognosis After LVAD Implantation. J Card Fail 2018;24:575-82.

73. Aaronson KD, Slaughter MS, Miller LW, et al. Use of an intrapericardial, continuous-flow, centrifugal pump in patients awaiting heart transplantation. Circulation 2012;125:3191-200.

74. Mehra MR, Uriel N, Naka Y, et al. A Fully Magnetically Levitated Left Ventricular Assist Device - Final Report. N Engl J Med 2019;380:1618-27.

75. Kormos RL, Teuteberg JJ, Pagani FD, et al. Right ventricular failure in patients with the HeartMate II continuous-flow left ventricular assist device: incidence, risk factors, and effect on outcomes. J Thorac Cardiovasc Surg 2010;139:1316-24.

76. LaRue SJ, Raymer DS, Pierce BR, et al. Clinical outcomes associated with INTERMACS-defined right heart failure after left ventricular assist device implantation. J Heart Lung Transplant 2017;36:475-7.

77. Kirklin JK, Pagani FD, Kormos RL, et al. Eighth annual INTERMACS report: Special focus on framing the impact of adverse events. J Heart Lung Transplant 2017;36:1080-6.

78. Joly JM, El-Dabh A, Kirklin JK, et al. High Right Atrial Pressure and Low Pulse Pressure Predict Gastrointestinal Bleeding in Patients With Left Ventricular Assist Device. J Card Fail 2018;24:487-93.

79. Sparrow CT, LaRue SJ, Schilling JD. Intersection of Pulmonary Hypertension and Right Ventricular Dysfunction in Patients on Left Ventricular Assist Device Support: Is There a Role for Pulmonary Vasodilators? Circ Heart Fail 2018;11:e004255.

80. Takeda K, Takayama H, Colombo PC, et al. Incidence and clinical significance of late right heart failure during continuous-flow left ventricular assist device support. J Heart Lung Transplant 2015;34:1024-32.

81. Takeda K, Takayama H, Colombo PC, et al. Late right heart failure during support with continuous-flow left ventricular assist devices adversely affects post-transplant outcome. J Heart Lung Transplant 2015;34:667-74.

82. Lampert BC, Teuteberg JJ. Right ventricular failure after left ventricular assist devices. J Heart Lung Transplant 2015;34:1123-30.

83. Topilsky Y, Hasin T, Oh JK, et al. Echocardiographic variables after left ventricular assist device implantation associated with adverse outcome. Circ Cardiovasc Imaging
2011;4:648-61.

84. Imamura T, Jeevanandam V, Kim G, et al. Optimal Hemodynamics During Left Ventricular Assist Device Support Are Associated With Reduced Readmission Rates. Circ Heart Fail 2019;12:e05094.

85. Shah P, Badoe N, Phillips S, et al. Unrecognized Left Heart Failure in LVAD Recipients: The Role of Routine Invasive Hemodynamic Testing. ASAIO J 2018;64:183-90.

86. Kirklin JK, Pagani FD, Goldstein DJ, et al. American Association for Thoracic Surgery/International Society for Heart and Lung Transplantation guidelines on selected topics in mechanical circulatory support. J Thorac Cardiovasc Surg 2020;159:865-96.

87. Hamdan R, Mansour H, Nassar P, et al. Prevention of right heart failure after left ventricular assist device implantation by phosphodiesterase 5 inhibitor. Artif Organs 2014;38:963-7.

88. Ishihara S, Kioka H, Ohtani T, et al. Successful treatment of severe combined post- and pre-capillary pulmonary hypertension in a patient with idiopathic restrictive cardiomyopathy. Pulm Circ 2018;8:2045894018770131.

89. Gulati G, Grandin EW, Kennedy K, et al. Preimplant Phosphodiesterase-5 Inhibitor Use Is Associated With Higher Rates of Severe Early Right Heart Failure After Left Ventricular Assist Device Implantation. Circ Heart Fail 2019;12:e005537.

90. Ichinose F, Roberts JD, Jr., Zapol WM. Inhaled nitric oxide: a selective pulmonary vasodilator: current uses and therapeutic potential. Circulation 2004;109:3106-11.

91. Chang JC, Sawa Y, Ohtake S, et al. Hemodynamic effect of inhaled nitric oxide in dilated cardiomyopathy patients on LVAD support. ASAIO J 1997;43:M418-21.

92. Wagner F, Dandel M, Gunther G, et al. Nitric oxide inhalation in the treatment of right ventricular dysfunction following left ventricular assist device implantation. Circulation 1997;96:II-291-6.

93. Potapov E, Meyer D, Swaminathan M, et al. Inhaled nitric oxide after left ventricular assist device implantation: a prospective, randomized, double-blind, multicenter, placebo-controlled trial. J Heart Lung Transplant 2011;30:870-8.

94. Argenziano M, Choudhri AF, Moazami N, et al. Randomized, double-blind trial of inhaled nitric oxide in LVAD recipients with pulmonary hypertension. Ann Thorac Surg 1998;65:340-5.

95. Mychaskiw G, Sachdev V, Heath BJ. Sildenafil (viagra) facilitates weaning of inhaled nitric oxide following placement of a biventricular-assist device. J Clin Anesth 
2001;13:218-20.

96. Groves DS, Blum FE, Huffmyer JL, et al. Effects of early inhaled epoprostenol therapy on pulmonary artery pressure and blood loss during LVAD placement. J Cardiothorac Vasc Anesth 2014;28:652-60.

97. Antoniou T, Prokakis C, Athanasopoulos G, et al. Inhaled nitric oxide plus iloprost in the setting of post-left assist device right heart dysfunction. Ann Thorac Surg 2012;94:792-8.

98. Tariq S, Aronow WS. Use of Inotropic Agents in Treatment of Systolic Heart Failure. Int J Mol Sci 2015;16:29060-8.

99. Levy JH, Bailey JM, Deeb GM. Intravenous milrinone in cardiac surgery. Ann Thorac Surg 2002;73:325-30.

100. Feneck RO. Milrinone and postoperative pulmonary hypertension. J Cardiothorac Vasc Anesth 1993;7:21-3.

101.Jeon Y, Ryu JH, Lim YJ, et al. Comparative hemodynamic effects of vasopressin and norepinephrine after milrinoneinduced hypotension in off-pump coronary artery bypass surgical patients. Eur J Cardiothorac Surg 2006;29:952-6.

102. Haglund NA, Burdorf A, Jones T, et al. Inhaled Milrinone After Left Ventricular Assist Device Implantation. J Card Fail 2015;21:792-7.

103. Feldman D, Pamboukian SV, Teuteberg JJ, et al. The 2013 International Society for Heart and Lung Transplantation Guidelines for mechanical circulatory support: executive summary. J Heart Lung Transplant 2013;32:157-87.

Cite this article as: Pirlamarla P, Rame E, Hoopes C, Rajapreyar I. Pulmonary vasodilator use in continuous-flow left ventricular assist device management. Ann Transl Med 2021;9(6):522. doi: 10.21037/atm-20-4710
104. Corbin JD, Francis SH. Cyclic GMP phosphodiesterase-5: target of sildenafil. J Biol Chem 1999;274:13729-32.

105. Nagendran J, Archer SL, Soliman D, et al. Phosphodiesterase type 5 is highly expressed in the hypertrophied human right ventricle, and acute inhibition of phosphodiesterase type 5 improves contractility. Circulation 2007;116:238-48.

106. Tedford RJ, Hemnes AR, Russell SD, et al. PDE5A inhibitor treatment of persistent pulmonary hypertension after mechanical circulatory support. Circ Heart Fail 2008;1:213-9.

107. Roberts KL, Shuster JE, Britt NS, et al. Evaluation of Clinical Outcomes with Phosphodiesterase-5 Inhibitor Therapy for Right Ventricular Dysfunction After Left Ventricular Assist Device Implantation. ASAIO J 2019;65:264-9.

108. Saeed O, Rangasamy S, Selevany I, et al. Sildenafil Is Associated With Reduced Device Thrombosis and Ischemic Stroke Despite Low-Level Hemolysis on Heart Mate II Support. Circ Heart Fail 2017;10.

109. Ravichandran AK, LaRue SJ, Novak E, et al. Sildenafil in Left Ventricular Assist Device Is Safe and Well-Tolerated. ASAIO J 2018;64:280-1.

110.LaRue SJ, Garcia-Cortes R, Nassif ME, et al. Treatment of secondary pulmonary hypertension with bosentan after left ventricular assist device implantation. Cardiovasc Ther 2015;33:50-5. 